\title{
A TWO-DIMENSIONAL SLICE THROUGH THE PARAMETER SPACE OF TWO-GENERATOR KLEINIAN GROUPS
}

\author{
ELENA KLIMENKO AND NATALIA KOPTEVA
}

\begin{abstract}
We describe all real points of the parameter space of two-generator Kleinian groups with a parabolic generator, that is, we describe a certain twodimensional slice through this space. In order to do this we gather together known discreteness criteria for two-generator groups and present them in the form of conditions on parameters. We complete the description by giving discreteness criteria for groups generated by a parabolic and a $\pi$-loxodromic elements whose commutator has real trace and present all orbifolds uniformized by such groups.
\end{abstract}

\section{INTRODUCTION}

A two-generator subgroup $\Gamma=\langle f, g\rangle$ of $\operatorname{PSL}(2, \mathbb{C})$ is determined up to conjugacy by its parameters $\beta=\beta(f)=\operatorname{tr}^{2} f-4, \beta^{\prime}=\beta(g)=\operatorname{tr}^{2} g-4$, and $\gamma=\gamma(f, g)=$ $\operatorname{tr}[f, g]-2$ whenever $\gamma \neq 0$ [6]. So the conjugacy class of an ordered pair $\{f, g\}$ can be identified with a point in the parameter space $\mathbb{C}^{3}=\left\{\left(\beta, \beta^{\prime}, \gamma\right)\right\}$ whenever $\gamma \neq 0$. The subspace $\mathcal{K}$ of $\mathbb{C}^{3}$ that corresponds to the discrete non-elementary groups $\Gamma=\langle f, g\rangle$ is called the parameter space of two-generator Kleinian groups. Note that a two-generator Kleinian group $\Gamma$ can be represented by several points in $\mathcal{K}$, since the same group can have different generating pairs.

Among all two-generator subgroups of $\operatorname{PSL}(2, \mathbb{C})$, we distinguish the class of $\mathcal{R} \mathcal{P}$ groups (two-generator groups with real parameters):

$$
\mathcal{R P}=\left\{\Gamma: \Gamma=\langle f, g\rangle \text { for some } f, g \in \operatorname{PSL}(2, \mathbb{C}) \text { with }\left(\beta, \beta^{\prime}, \gamma\right) \in \mathbb{R}^{3}\right\} .
$$

The aim of this paper is to completely determine all points in $\mathbb{C}^{3}$ that are parameters for the discrete non-elementary $\mathcal{R} \mathcal{P}$ groups with one generator parabolic:

$$
S_{\infty}=\{(\gamma, \beta):(\beta, 0, \gamma) \text { are parameters for some }\langle f, g\rangle \in \mathcal{D} \mathcal{R} \mathcal{P}\},
$$

where $\mathcal{D} \mathcal{R} \mathcal{P}$ denotes the class of all discrete non-elementary $\mathcal{R} \mathcal{P}$ groups. Geometrically, $S_{\infty}$ is a two-dimensional slice through the six-dimensional parameter space $\mathcal{K}$.

The slice $S_{\infty}$ intersects the well-known Riley slice $(0,0, \gamma), \gamma \in \mathbb{C}$, which consists of all Kleinian groups generated by two parabolics.

Consider the sequence of slices $\left\{S_{n}\right\}_{n=2}^{\infty}$, where

$$
S_{n}=\left\{(\gamma, \beta):\left(\beta,-4 \sin ^{2}(\pi / n), \gamma\right) \text { are parameters for some }\langle f, g\rangle \in \mathcal{D} \mathcal{R} \mathcal{P}\right\} .
$$

Date: July 28, 2018.

1991 Mathematics Subject Classification. Primary: 30F40; Secondary: 20H10, 22E40, 57M60.

Key words and phrases. Kleinian group, discrete group, hyperbolic orbifold.

The first author was supported by Gettysburg College Research and Professional Development Grant, 2005-2006. The research of the second author was supported by FP6 Marie Curie IIF Fellowship and carried out at LATP (UMR CNRS 6632). 
The first slice $S_{2}$ of this sequence is of great interest in the theory of discrete groups. This slice consists of all parameters for discrete $\mathcal{R} \mathcal{P}$ groups with an elliptic generator of order 2 and was investigated in [5]. It was shown that if $\langle f, g\rangle$ has parameters $\left(\beta, \beta^{\prime}, \gamma\right)$, then there exists a group $\langle f, h\rangle$ with parameters $(\beta,-4, \gamma)$ such that if $\gamma \neq 0, \beta$, then $\langle f, h\rangle$ is discrete whenever $\langle f, g\rangle$ is. Hence, the slice $S_{2}$ gives necessary discreteness conditions for a group with parameters $\left(\beta, \beta^{\prime}, \gamma\right)$, where $\beta$ and $\gamma$ are real. It follows that every $S_{n}$ with $n>2$, including $S_{\infty}$, is a subset of $S_{2}$.

Since a parabolic element can be viewed as the limit of a sequence of primitive elliptic elements of order $n$ as $n \rightarrow \infty$, the following two questions for $\left\{S_{n}\right\}$ and $S_{\infty}$ naturally arise.

(1) Is it true that for every point $x \in S_{\infty}$ there exists a sequence $\left\{x_{k}\right\}_{k=2}^{\infty}$ with $x_{k} \in S_{k}$ that converges to $x$ ?

(2) Is it true that for each $\varepsilon>0$ there exists $N \in \mathbb{N}$ such that the $\varepsilon$ neighbourhood of $S_{\infty}$ contains $S_{n}$ for all $n>N$ ?

Note that the structure of $S_{n}$ for $n>2$ is unknown.

We work out $S_{\infty}$ by splitting the plane $(\gamma, \beta)$ into several parts. It turns out that $\Gamma=\langle f, g\rangle$ has an invariant plane in one of the following cases: (1) $\gamma<0$ and $\beta \leq-4$; (2) $\gamma>0$ and $\beta \geq-4$. Such discrete groups were investigated, for example, in [13] and 8, 14, 15, respectively. If $\gamma<0$ and $\beta>-4$, then $\Gamma$ is truly spatial (non-elementary and without invariant plane) and this case is treated in [11. We get these dicreteness criteria together and transform them into conditions on $\beta$ and $\gamma$ if it was not done before.

So the last case to consider is when $\gamma>0$ and $\beta<-4$. In this case $\Gamma$ is truly spatial with $f \pi$-loxodromic. We complete the study of the slice $S_{\infty}$ by giving discreteness criteria for such groups.

The paper is organised as follows. In Section 2, discreteness criteria are given for truly spatial $\mathcal{R P}$ groups $\Gamma$ generated by a $\pi$-loxodromic and a parabolic elements (Theorems 2.1 and 2.6). In Section 3, for each such discrete $\Gamma$ we obtain a presentation and the Kleinian orbifold $Q(\Gamma)$ (Theorem 3.1). Section 4 is devoted to the analysis of the parameter space. We completely describe the slice $S_{\infty}$ by giving explicit formulas for the parameters $\beta$ and $\gamma$. We also program the obtained formulas in the package Maple 7.0 and plot a part of $S_{\infty}$ on the $(\gamma, \beta)$-plane to give an idea of how it looks like.

\section{DisCRETENESS CRITERIA}

Recall that an element $f \in \operatorname{PSL}(2, \mathbb{C})$ with real $\beta(f)$ is elliptic, parabolic, hyperbolic, or $\pi$-loxodromic according to whether $\beta(f) \in[-4,0), \beta(f)=0$, $\beta(f) \in(0,+\infty)$, or $\beta(f) \in(-\infty,-4)$. If $\beta(f) \notin[-4,+\infty)$, then $f$ is called strictly loxodromic.

An elliptic element $f$ of order $n$ is said to be non-primitive if it is a rotation through $2 \pi q / n$, where $q$ and $n$ are coprime $(1<q<n / 2)$. If $f$ is a rotation through $2 \pi / n$, then it is called primitive.

Theorem 2.1. Let $f \in \operatorname{PSL}(2, \mathbb{C})$ be a $\pi$-loxodromic element, $g \in \operatorname{PSL}(2, \mathbb{C})$ be a parabolic element, and let $\Gamma=\langle f, g\rangle$ be a non-elementary $\mathcal{R} \mathcal{P}$ group without invariant plane. Then 
(1) there exist unique elements $h_{1}, h_{2} \in \operatorname{PSL}(2, \mathbb{C})$ such that $h_{1}^{2}=f g^{-1} f^{-1} g^{-1}$ and $\left(h_{1} g\right)^{2}=1, h_{2}^{2}=f^{-1} g^{-1} f^{2} g f^{-1}$ and $\left(h_{2} f g^{-1} f^{-1}\right)^{2}=1$.

(2) the group $\Gamma$ is discrete if and only if one of the following conditions holds:

(i) $h_{1}$ is either a hyperbolic, or parabolic, or primitive elliptic element of even order $m \geq 4$, and $h_{2}$ is either a hyperbolic, or parabolic, or primitive elliptic element of order $p \geq 3$;

(ii) $h_{1}$ is a primitive elliptic element of odd order $m \geq 3$, and $h_{2} h_{1}$ is either a hyperbolic, or parabolic, or primitive elliptic element of order $k \geq 3$.

Basic geometric construction. We will construct a group $\Gamma^{*}$ that contains $\Gamma=$ $\langle f, g\rangle$ as a subgroup of finite index. The idea is to find $\Gamma^{*}$ so that a fundamental polyhedron for a discrete $\Gamma^{*}$ can be easily constructed. It will be clear from the construction that $\Gamma$ is commensurable with a reflection group which either coincides with $\Gamma^{*}$ or is an index 2 subgroup of $\Gamma^{*}$. The construction presented below will be used throughout Sections 2 and 3 and we shall use the notation introduced here.

Let $f$ and $g$ be as in the statement of Theorem 2.1 Since $\Gamma$ is a non-elementary $\mathcal{R} \mathcal{P}$ group without invariant plane, there exists an invariant plane of $g$, say $\eta$, which is orthogonal to the axis of $f$ [9, Theorem 2].

Denote by $M$ the fixed point of $g$ and by $\omega$ the plane that passes through $M$ and $f$ (we denote elements and their axes by the same letters when it does not lead to any confusion). Note that $f$ keeps $\omega$ invariant. Since $f$ is orthogonal to $\eta, \omega$ is also orthogonal to $\eta$. Let $e$ be the half-turn with the axis $\omega \cap \eta$. Then $e$ passes through $M$ and is orthogonal to $f$.

Let $e_{f}$ and $e_{g}$ be half-turns such that

$$
f=e_{f} e \text { and } g=e_{g} e .
$$

Then $e_{f}$ is orthogonal to $\omega$ and $e_{g}$ lies in $\eta$.

Let $\tau$ be the plane passing through $e_{g}$ orthogonally to $\eta$ and let $\sigma=e_{f}(\tau)$. The planes $\tau$ and $\omega$ are parallel and $M$ is their common point on the boundary $\partial \mathbb{H}^{3}$. Since $e_{f}$ is orthogonal to $\omega$, the planes $\sigma$ and $\omega$ are also parallel with the common point $e_{f}(M)$ on $\partial \mathbb{H}^{3}$. Since $e_{f}(M) \neq M$, the planes $\omega, \sigma$, and $\tau$ do not have a common point in $\overline{\mathbb{H}^{3}}=\mathbb{H}^{3} \cup \partial \mathbb{H}^{3}$. Therefore, there exists a unique plane $\delta$ orthogonal to all $\omega, \sigma$, and $\tau$. It is clear that $e_{f} \subset \delta$.

Consider two extensions of $\Gamma: \widetilde{\Gamma}=\langle f, g, e\rangle$ and $\Gamma^{*}=\left\langle f, g, e, R_{\omega}\right\rangle$. (We denote the reflection in a plane $\kappa$ by $R_{\kappa}$.) One can show that $\widetilde{\Gamma}=\left\langle e_{f}, e_{g}, e\right\rangle$ and $\Gamma^{*}=$ $\left\langle e_{f}, R_{\eta}, R_{\omega}, R_{\tau}\right\rangle$. From (2.1), it follows that $\widetilde{\Gamma}$ contains $\Gamma$ as a subgroup of index at most 2. Moreover, $\widetilde{\Gamma}$ is the orientation preserving subgroup of $\Gamma^{*}$ and, hence, $\Gamma^{*}$ contains $\Gamma$ as a subgroup of finite index. Therefore, $\Gamma, \widetilde{\Gamma}$, and $\Gamma^{*}$ are either all discrete, or all non-discrete. We then concentrate on the group $\Gamma^{*}$.

Let $\mathcal{P}^{*}$ be the infinite volume polyhedron bounded by $\eta, \omega, \tau, \sigma$, and $\delta$. $\mathcal{P}^{*}$ has five right dihedral angles (between faces lying in $\eta$ and $\omega, \eta$ and $\tau, \delta$ and $\omega, \delta$ and $\tau$, and $\delta$ and $\sigma$ ). The plane $\sigma$ may either intersect with, or be parallel to, or be disjoint from each of $\tau$ and $\eta$.

If $\sigma$ and $\tau$ intersect, then we denote the dihedral angle of $\mathcal{P}^{*}$ between them by $2 \pi / m$, where $m>2, m$ is not necessary an integer. We keep the notation $2 \pi / m$ taking $m=\infty$ and $m=\bar{\infty}$ for parallel or disjoint $\sigma$ and $\tau$, respectively. Similarly, we denote the "dihedral angle" between $\eta$ and $\sigma$ by $\pi / p$, where $p>2$ is real, $\infty$, 


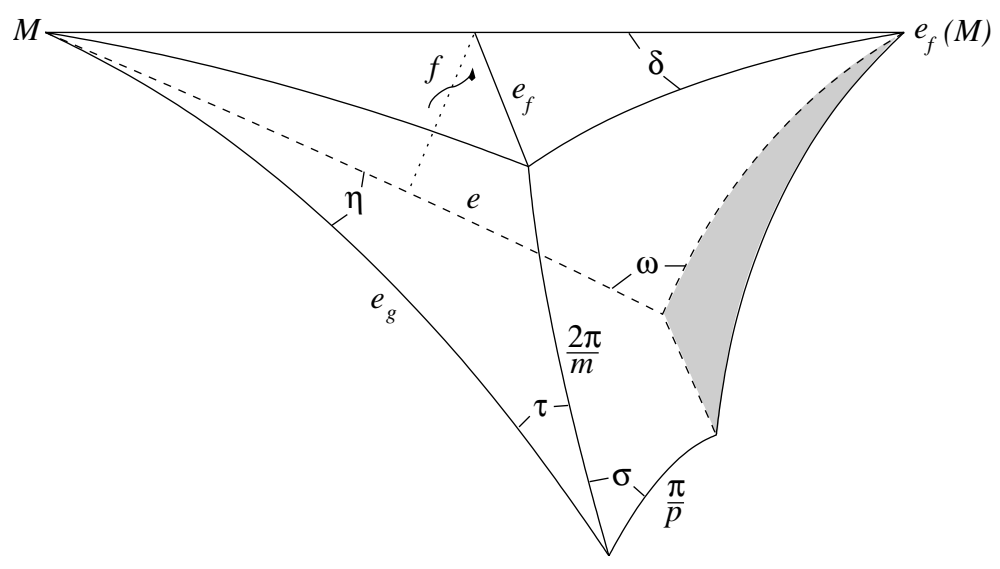

Figure 1. Polyhedron $\mathcal{P}^{*}$

or $\bar{\infty}$. (We regard $\bar{\infty}>\infty>x, x / \infty=x / \bar{\infty}=0, \infty / x=\infty, \bar{\infty} / x=\bar{\infty}$ for any positive real $x$.) $\mathcal{P}^{*}$ exists in $\mathbb{H}^{3}$ for all $m>2$ and $p>2$ by [16].

In Figure 1 $\mathcal{P}^{*}$ is drawn under assumption that $m<\infty, p<\infty$, and $1 / 2+$ $1 / p+2 / m>1$. The shaded triangle shows the hyperbolic plane orthogonal to $\eta, \sigma$, and $\omega$. Note that this plane is not a face of $\mathcal{P}^{*}$ and is shown only to underline the combinatorial structure of $\mathcal{P}^{*}$. In figures, we do not label dihedral angles of $\pi / 2$ in order to not overload the picture.

Suppose now that $m<\infty$, that is $\sigma$ and $\tau$ intersect. Let $\xi$ be the plane passing through $e_{f}$ orthogonally to $\delta$. Then $\xi$ is orthogonal to $\omega$. One can see that $\sigma=$ $R_{\xi}(\tau)$ and $\xi$ is the bisector of the dihedral angle of $\mathcal{P}^{*}$ made by $\tau$ and $\sigma$.

Let $\mathcal{Q}^{*}$ be the polyhedron bounded by $\eta, \tau, \omega, \delta$, and $\xi$. $\mathcal{Q}^{*}$ has six dihedral angles of $\pi / 2$; the dihedral angle between $\tau$ and $\xi$ is equal to $\pi / m$ with $2<m<\infty$. Denote the "dihedral angle" between $\eta$ and $\xi$ by $\pi / k$, where $k>2$ is real, $k=\infty$, or $k=\bar{\infty}$. $\mathcal{Q}^{*}$ exists in $\mathbb{H}^{3}$ for all $m>2$ and $k>2$ by [16. Note that $R_{\xi}$ is not necessary in $\Gamma^{*}$, but if it is and if $\Gamma^{*}$ is discrete, then we will see that $\mathcal{Q}^{*}$ is a fundamental polyhedron for $\Gamma^{*}$. In Figure $2 \mathcal{Q}^{*}$ is drawn under assumption that $1 / 2+1 / k+1 / m>1$.

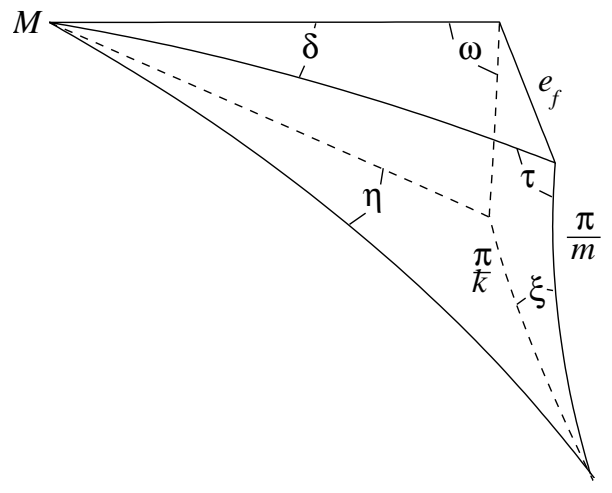

Figure 2. Polyhedron $\mathcal{Q}^{*}$ 
Lemma 2.2. Let $f \in \operatorname{PSL}(2, \mathbb{C})$ be a $\pi$-loxodromic element, $g \in \operatorname{PSL}(2, \mathbb{C})$ be a parabolic element, and let $\Gamma=\langle f, g\rangle$ be a non-elementary $\mathcal{R} \mathcal{P}$ group without invariant plane. Then there exist unique elements $h_{1}, h_{2} \in \operatorname{PSL}(2, \mathbb{C})$ such that

(1) $h_{1}^{2}=f g^{-1} f^{-1} g^{-1}$ and $\left(h_{1} g\right)^{2}=1$,

(2) $h_{2}^{2}=f^{-1} g^{-1} f^{2} g f^{-1}$ and $\left(h_{2} f g^{-1} f^{-1}\right)^{2}=1$.

Moreover, the elements $h_{1}$ and $h_{2}$ are not strictly loxodromic.

Proof. First, note that $R_{\sigma}=e_{f} R_{\tau} e_{f}$ and $g=R_{\tau} R_{\omega}$. Therefore,

$$
R_{\sigma} R_{\omega}=e_{f} R_{\tau} e_{f} R_{\omega}=e_{f} R_{\tau} R_{\omega} e_{f}=e_{f} g e_{f}=f g^{-1} f^{-1} .
$$

Let us show that if we take $h_{1}=R_{\xi} R_{\tau}=R_{\sigma} R_{\xi}$, then the assertion (1) of the lemma hold. Indeed,

$$
h_{1}^{2}=R_{\sigma} R_{\tau}=\left(R_{\sigma} R_{\omega}\right)\left(R_{\omega} R_{\tau}\right)=f g^{-1} f^{-1} g^{-1} .
$$

Moreover, $h_{1} g=\left(R_{\xi} R_{\tau}\right)\left(R_{\tau} R_{\omega}\right)=R_{\xi} R_{\omega}$. Since $\xi$ and $\omega$ are orthogonal, $\left(R_{\xi} R_{\omega}\right)^{2}=1$. Hence, $\left(h_{1} g\right)^{2}=1$. Note also that since $h_{1}$ is a product of two reflections, $h_{1}$ is not strictly loxodromic.

Now let us show that $h_{1}$ is unique. The element $f g^{-1} f^{-1} g^{-1}$ is uniquely determined as an element of $\operatorname{PSL}(2, \mathbb{C})$.

If $f g^{-1} f^{-1} g^{-1}$ is parabolic, it has only one square root $h_{1}$. Suppose that $f g^{-1} f^{-1} g^{-1}$ is hyperbolic. Then it has exactly two square roots, one of which is $h_{1}$ defined above and the other, denoted $\bar{h}_{1}$, is a $\pi$-loxodromic element with the same axis and translation length as $h_{1}$. Clearly, $\left(\bar{h}_{1} g\right)^{2} \neq 1$.

If $f g^{-1} f^{-1} g^{-1}$ is elliptic, then it also has two square roots $h_{1}$ and $\bar{h}_{1}$, both are elliptic elements. The element $\bar{h}_{1}$ is elliptic with the same axis as $h_{1}$ and with rotation angle $(\pi-2 \pi / m)$, while $h_{1}$ is a rotation through $2 \pi / m$ in the opposite direction. Again, $\left(\bar{h}_{1} g\right)^{2} \neq 1$.

Now we take

$$
h_{2}=R_{\eta} R_{\sigma}=\left(R_{\eta} R_{\tau}\right)\left(R_{\tau} R_{\sigma}\right)=e_{g} h_{1}^{-2}=e f g f^{-1} .
$$

Then

$$
h_{2}^{2}=f^{-1} g^{-1} f^{2} g f^{-1} \quad \text { and } \quad\left(f g^{-1} f^{-1} h_{2}\right)^{2}=1 .
$$

These two conditions determine $h_{2}$ uniquely.

Note that the elements $h_{1}, h_{2}$ defined in Lemma 2.2 determine combinatorial and metric structures of $\mathcal{P}^{*}$. For example, if $h_{1}$ is elliptic, then its rotation angle is equal to the dihedral angle of $\mathcal{P}^{*}$ between $\sigma$ and $\tau$. If $h_{2}$ is elliptic, then its rotation angle is equal to the doubled dihedral angle of $\mathcal{P}^{*}$ between $\eta$ and $\sigma$. Vice versa, if the metric structure of $\mathcal{P}^{*}$ is fixed, then the types of elements $h_{1}$ and $h_{2}$ can be determined.

The same can be said about $\mathcal{Q}^{*}$ and the elements $h_{1}$ and $h_{2} h_{1}$. The element $h_{2} h_{1}$ is responsible for the mutual position of the planes $\eta$ and $\xi$ (see the proof of Lemma 2.5).

Lemmas 2.3 2.5 below give some necessary conditions for discreteness of $\Gamma$ via conditions on elements $h_{1}$ and $h_{2}$. One needs to keep in mind the connection between these elements and the polyhedra $\mathcal{P}^{*}$ and $\mathcal{Q}^{*}$.

Lemma 2.3. If $\Gamma$ is discrete, then $h_{1}$ is either a hyperbolic, or parabolic, or primitive elliptic element of order $m \geq 3$. 
Proof. The subgroup $H=\left\langle g, f g f^{-1}\right\rangle$ of $\Gamma$ keeps $\delta$ invariant and is conjugate to a subgroup of $\operatorname{PSL}(2, \mathbb{R})$. Since $\Gamma$ is discrete, $H$ must be discrete. By [15] or [2], the group $H$ is discrete if and only if either

(1) $f g^{-1} f^{-1} g^{-1}=h_{1}^{2}$ is a hyperbolic, or a parabolic, or a primitive elliptic element, or

(2) $h_{1}$ is a primitive elliptic element of odd order $m$, where $m \geq 3$.

If $h_{1}^{2}$ is parabolic of hyperbolic, then $h_{1}$ is parabolic or hyperbolic, respectively. If $h_{1}^{2}$ is a primitive elliptic element, then $h_{1}$ is a primitive elliptic of even order $m \geq 4$.

Lemma 2.4. If $\Gamma$ is discrete, then $h_{2}$ is either a hyperbolic, or parabolic, or primitive elliptic element of order $p \geq 3$.

Proof. Let $\kappa$ be the plane orthogonal to $\eta, \sigma$, and $\omega$. The subgroup $H=\left\langle e, f g f^{-1}\right\rangle$ of $\widetilde{\Gamma}$ keeps the plane $\kappa$ invariant and is conjugate to a subgroup of $\operatorname{PSL}(2, \mathbb{R})$. By [15], $H$ is discrete if and only if $h_{2}=e f g f^{-1}$ is either a hyperbolic, or parabolic, or primitive elliptic element of order $p \geq 3$.

Lemma 2.5. If $\Gamma$ is discrete and $h_{1}$ is a primitive elliptic element of odd order, then $h_{2} h_{1}$ is either a hyperbolic, or parabolic, or primitive elliptic element of order $k \geq 3$.

Proof. Recall that $\Gamma^{*}=\left\langle e_{f}, R_{\eta}, R_{\tau}, R_{\omega}\right\rangle$. Since $h_{1}$ has odd order and $h_{1}^{2} \in \Gamma^{*}$, $h_{1} \in \Gamma^{*}$. Since, moreover, $h_{1}=R_{\xi} R_{\tau}, e_{f}=R_{\delta} R_{\xi}$, and $R_{\tau} \in \Gamma^{*}$, both $R_{\xi}$ and $R_{\delta}$ are also in $\Gamma^{*}$. Further, since the plane $\xi$ is orthogonal to $\omega$, the group $\left\langle R_{\eta} R_{\delta}, e_{f}\right\rangle$ keeps $\omega$ invariant and is conjugate to a subgroup of $\operatorname{PSL}(2, \mathbb{R})$. It is clear that $\left\langle R_{\eta} R_{\delta}, e_{f}\right\rangle$ is discrete if and only if $R_{\eta} R_{\xi}=h_{2} h_{1}$ is a hyperbolic, parabolic, or primitive elliptic element of order $k \geq 3$ [15].

Proof of Theorem 2.1. Lemma 2.2 proves existence and uniqueness of elements $h_{1}$ and $h_{2}$. Now we prove part (2) of the theorem.

If $\Gamma$ is discrete then $h_{1}$ is either a hyperbolic, or parabolic, or primitive elliptic element of order $m \geq 3$ by Lemma 2.3 . We split the discrete groups $\Gamma$ into two families. The first family consists of those groups for which $h_{1}$ is hyperbolic, parabolic, or primitive elliptic of even order. By Lemma 2.4 for these groups $h_{2}$ is a hyperbolic, parabolic, or primitive elliptic element.

The second family consists of the discrete groups with $h_{1}$ elliptic of odd order. Then by Lemma 2.5 $h_{2} h_{1}$ is a hyperbolic, or parabolic, or primitive elliptic element of order $k \geq 3$. (Note that in this case $h_{2}$ is necessarily hyperbolic or primitive elliptic.)

So if $\Gamma$ is discrete, then either (2)(i) or (2)(ii) of Theorem 2.1 can occur. Clearly, if neither (2)(i) nor (2)(ii) holds, then $\Gamma$ is not discrete by Lemmas 2.3 2.5

Now prove that each of (2)(i) and (2)(ii) is a sufficient condition for $\Gamma$ to be discrete. In each of the two cases we will give a fundamental polyhedron for $\Gamma^{*}$ to show, by using the Poincaré polyhedron theorem [3], that $\Gamma^{*}$ is discrete.

Suppose that (2)(i) holds. Then since $m$ is even, the group $G_{1}$ generated by the side pairing transformations $R_{\eta}, R_{\omega}, R_{\sigma}, R_{\tau}$, and $e_{f}$ and the polyhedron $\mathcal{P}^{*}$ satisfy the Poincaré polyhedron theorem, $G_{1}$ is discrete and $\mathcal{P}^{*}$ is its fundamental polyhedron. Obviously, $G_{1}=\Gamma^{*}$. 
Suppose that (2)(ii) holds. Then the group $G_{2}$ generated by the side pairing transformations $R_{\eta}, R_{\omega}, R_{\xi}, R_{\tau}$, and $R_{\delta}$ and the polyhedron $\mathcal{Q}^{*}$ satisfy the Poincaré theorem, $G_{2}$ is discrete, and $\mathcal{Q}^{*}$ is its fundamental polyhedron.

In the proof of Lemma 2.5] it was shown that, for $m$ odd, $R_{\xi} \in \Gamma^{*}$ and $R_{\delta} \in \Gamma^{*}$. Moreover, $e_{f}=R_{\xi} R_{\delta}$. Hence, $G_{2}=\Gamma^{*}$, so $\Gamma^{*}$ is discrete.

Theorem 2.1] is proved.

Our next goal is to compute parameters $(\beta(f), \beta(g), \gamma(f, g))$ for both series of discrete groups listed in Theorem 2.1]

If $f \in \operatorname{PSL}(2, \mathbb{C})$ is a loxodromic element with translation length $d_{f}$ and rotation angle $\theta_{f}$, then

$$
\operatorname{tr}^{2} f=4 \cosh ^{2} \frac{d_{f}+i \theta_{f}}{2}
$$

and $\lambda_{f}=d_{f}+i \theta_{f}$ is called the complex translation length of $f$.

Note that if $f$ is hyperbolic then $\theta_{f}=0$ and $\operatorname{tr}^{2} f=4 \cosh ^{2}\left(d_{f} / 2\right)$. If $f$ is elliptic then $d_{f}=0$ and $\operatorname{tr}^{2} f=4 \cos ^{2}\left(\theta_{f} / 2\right)$. If $f$ is parabolic then $\operatorname{tr}^{2} f=4$; by convention we set $d_{f}=\theta_{f}=0$.

We define the set

$$
\mathcal{U}=\{u: u=i \pi / p \text { for some } p \in \mathbb{Z}, p \geq 2\} \cup[0,+\infty) .
$$

In other words, the set $\mathcal{U}$ consists of all complex translation half-lengths $u=\lambda_{f} / 2$ for hyperbolic, parabolic, and primitive elliptic elements $f$. Furthermore, we define a function $t: \mathcal{U} \rightarrow\{2,3,4, \ldots\} \cup\{\infty, \bar{\infty}\}$ as follows:

$$
t(u)=\left\{\begin{array}{lll}
p & \text { if } & u=i \pi / p \\
\infty & \text { if } & u=0 \\
\bar{\infty} & \text { if } & u \in(0,+\infty)
\end{array}\right.
$$

Given $u \in \mathcal{U}$ and $f$ with $\operatorname{tr}^{2} f=4 \cosh ^{2} u, t(u)$ determines the type of $f$ and, moreover, its order if $f$ is elliptic. Note also that since we regard $\infty / n=\infty$ and $\bar{\infty} / n=\bar{\infty}$, an expression of the form $(t(u), n)=1$ with $n>1$ means, in particular, that $t(u)$ is finite.

Theorem 2.6. Let $f, g \in \operatorname{PSL}(2, \mathbb{C})$ with $\beta(f)<-4, \beta(g)=0$, and $\gamma(f, g)>0$. Then $\Gamma=\langle f, g\rangle$ is discrete if and only if one of the following holds:

(1) $\gamma(f, g)=4 \cosh ^{2} u$ and $\beta(f)=-4 \cosh ^{2} v / \gamma(f, g)-4$, where $u, v \in \mathcal{U}$ with $t(u) \geq 4,(t(u), 2)=2$, and $t(v) \geq 3$;

(2) $\gamma(f, g)=4 \cosh ^{2} u$ and $\beta(f)=-4 \cosh ^{2} v-4$, where $u, v \in \mathcal{U}$ with $t(u) \geq 3$, $(t(u), 2)=1$, and $t(v) \geq 3$.

Proof. Obviously, $\beta(f)<-4$ and $\beta(g)=0$ if and only if $f$ is $\pi$-loxodromic and $g$ is parabolic. With this choice of $\beta(f)$ and $\beta(g), \gamma(f, g)>0$ if and only if the group $\Gamma=\langle f, g\rangle$ is a non-elementary $\mathcal{R} \mathcal{P}$ group without invariant plane 9. This means that the hypotheses of Theorem [2.6 are equivalent to the hypotheses of Theorem 2.1. Therefore, in order to prove Theorem 2.6 it is sufficient to calculate the parameters $\beta(f)$ and $\gamma(f, g)$ for both families of the discrete groups listed in Theorem 2.1

Let $\sigma^{\prime}$ be the image of $\sigma$ under $R_{\omega}$, that is $R_{\sigma^{\prime}}=R_{\omega} R_{\sigma} R_{\omega}$. Using the identity (2.2) and the fact that $g=R_{\tau} R_{\omega}$, we have

$$
[f, g]=f g f^{-1} g^{-1}=\left(R_{\omega} R_{\sigma}\right)\left(R_{\omega} R_{\tau}\right)=\left(R_{\sigma^{\prime}} R_{\omega}\right)\left(R_{\omega} R_{\tau}\right)=R_{\sigma^{\prime}} R_{\tau} .
$$


Note that $\sigma^{\prime}$ and $\tau$ are disjoint and $\delta$ is orthogonal to both of them. Therefore, $[f, g]$ is a hyperbolic element with the axis lying in $\delta$ and the translation length $2 d$, where $d$ is the distance between $\sigma^{\prime}$ and $\tau$. Hence, since $\gamma(f, g)>0$,

$$
\gamma(f, g)=\operatorname{tr}[f, g]-2=+2 \cosh d-2 .
$$

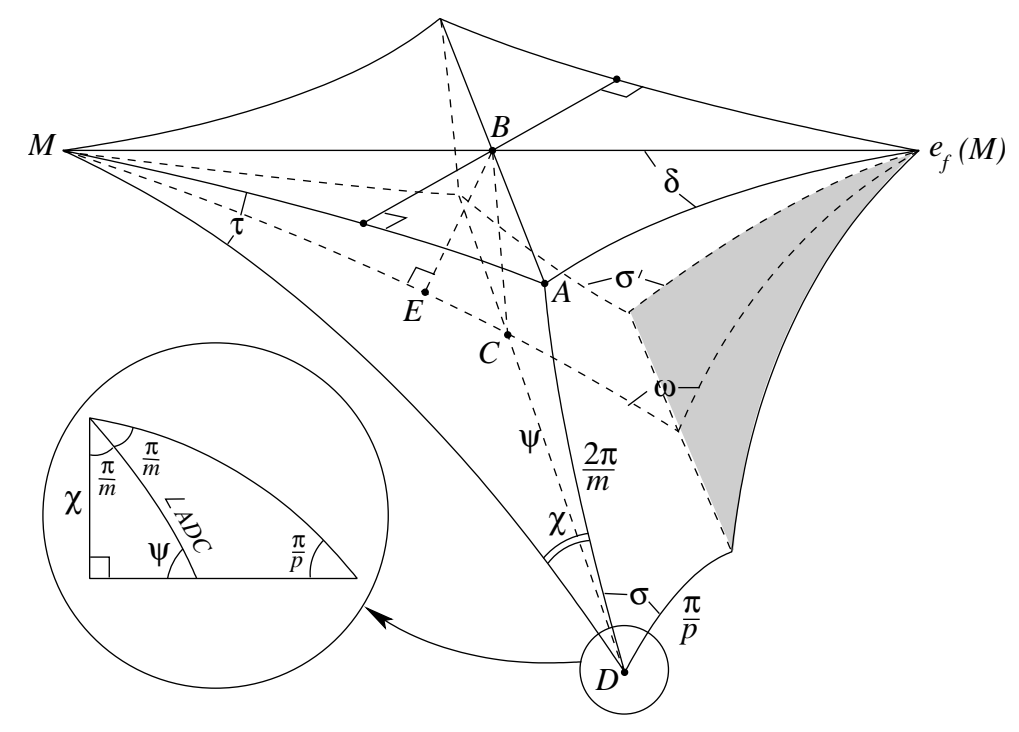

FiguRE 3.

Now, using generalised triangles in the plane $\delta$, it is not difficult to calculate that

$$
\gamma(f, g)= \begin{cases}4 \cos ^{2}(\pi / m) & \text { if } \quad 3 \leq m<\infty \\ 4 & \text { if } m=\infty \\ 4 \cosh ^{2}(d(\sigma, \tau) / 2) & \text { if } \quad m=\bar{\infty}\end{cases}
$$

where $d(\sigma, \tau)$ is the distance between $\sigma$ and $\tau$ if they are disjoint. Hence,

$$
\gamma(f, g)=4 \cosh ^{2} u
$$

where $u \in \mathcal{U}, t(u)=m \geq 3$.

Let us calculate $\beta(f)$. The element $f$ is $\pi$-loxodromic if and only $\operatorname{if}^{2} \operatorname{tr}^{2} f=$ $4 \cosh ^{2}(T+i \pi / 2)=-4 \sinh ^{2} T$, where $2 T$ is the translation length of $f$. That is,

$$
\beta(f)=-4 \sinh ^{2} T-4 .
$$

Note that $T$ is the distance between $e$ and $e_{f}$. It is measured in $\omega$ and equals $B E$ (see Figure 3).

Suppose that we are in case (2)(i) of Theorem 2.1 that is $(t(u), 2)=2$, and that $\sigma$ and $\tau$ intersect. Recall that $\xi$ is the bisector of the dihedral angle of $\mathcal{P}^{*}$ made by $\sigma$ and $\tau$. Let $\psi$ be the angle that $\xi$ makes with $\eta$. Note that $\psi=\angle B C E$. From the link of $D$, we have that

$$
\cos \chi=\frac{\cos (\pi / p)}{\sin (2 \pi / m)}=\frac{\cos \psi}{\sin (\pi / m)}
$$


and, therefore,

$$
\cos \psi=\frac{\cos (\pi / p)}{2 \cos (\pi / m)}
$$

Further, from the link of $D$,

$$
\cos \angle A D C=\frac{\cos \psi \cdot \cos (\pi / m)}{\sin \psi \cdot \sin (\pi / m)} .
$$

From the $\triangle A B M, \cosh ^{2} A B=1 / \sin (\pi / m)$ and, from the quadrilateral $A B C D$,

$$
\sinh B C=\frac{\cos \angle A D C}{\sinh A B}
$$

Finally, from $\triangle B C E$,

$$
\sinh T=\sinh B E=\sin \psi \cdot \sinh B C .
$$

Combining (2.3) - (2.6), we have that

$$
\sinh ^{2} T=\frac{\cos ^{2}(\pi / p)}{4 \cos ^{2}(\pi / m)}=\frac{\cos ^{2}(\pi / p)}{\gamma(f, g)} .
$$

Similar calculations can be done for parallel or disjoint $\sigma$ and $\tau$. Hence, $\beta(f)=$ $-\sinh ^{2} T-4=-\cosh ^{2} v / \gamma(f, g)-4$, where $v \in \mathcal{U}, t(v) \geq 3$.

Now note that in case (2)(ii) of Theorem 2.1 the angle $\psi=\angle B C E$ must be of the form $\pi / k, k \geq 3$ is an integer, $\infty$, or $\bar{\infty}$. Then we need to recompute the formulas (2.4)-(2.6) with $\psi=\pi / k$ :

$$
\cos \angle A D C=\frac{\cos (\pi / k) \cdot \cos (\pi / m)}{\sin (\pi / k) \cdot \sin (\pi / m)}, \quad \sinh B C=\frac{\cos \phi}{\sinh a}=\frac{\cos (\pi / k)}{\sin (\pi / k)} .
$$

Then

$$
\sinh T=\sin \psi \cdot \sinh B C=\cos (\pi / k) .
$$

Hence, $\beta(f)=-4 \cosh ^{2} v-4$, where $v \in \mathcal{U}, t(v) \geq 3$.

\section{ORBIFOLDS}

Denote by $\Omega(\Gamma)$ the discontinuity set of a Kleinian group $\Gamma$. The Kleinian orbifold $Q(\Gamma)=\left(\mathbb{H}^{3} \cup \Omega(\Gamma)\right) / \Gamma$ is said to be an orientable 3-orbifold with a complete hyperbolic structure on its interior $\mathbb{H}^{3} / \Gamma$ and a conformal structure on its boundary $\Omega(\Gamma) / \Gamma$.

We need the following (Kleinian) group presentations:

- $P H[\infty, m ; q]=\langle x, y, s| x^{\infty}=s^{2}=(x s)^{2}=(y s)^{2}=\left(x y x y^{-1}\right)^{m}=$ $\left.\left(y^{-1} x y s\right)^{q}=1\right\rangle$

- $P[\infty, m ; q]=\langle w, x, y, z| w^{\infty}=x^{2}=y^{2}=z^{2}=(w x)^{2}=(w y)^{2}=(y z)^{2}=$ $\left.(z x)^{q}=(z w)^{m}=1\right\rangle$

- $\mathcal{S}_{2}[\infty, m ; q]=\left\langle x, L \mid x^{\infty}=\left(x L x L^{-1}\right)^{m}=\left(x L^{2} x^{-1} L^{-2}\right)^{q}=1\right\rangle$,

- $\operatorname{GTet}_{1}[\infty, m ; q]=\langle x, y, z| x^{\infty}=y^{2}=z^{\infty}=(x y)^{m}=\left(y z y^{-1} z^{-1}\right)^{q}=$ $[x, z]=1\rangle$.

Here $m$ and $q$ are integers greater than 1 , or $\infty$ or $\bar{\infty}$ with the following convention. If we have a relation of the form $w^{n}=1$ with $n=\bar{\infty}$, then we simply remove the relation $w^{n}=1$ from the presentation (in fact, this means that the element $w$ is hyperbolic). Further, if $n=\infty$ and we keep the relation $w^{n}=1 \sim w^{\infty}=1$, we get a Kleinian group presentation where parabolics are indicated. To get an abstract group presentation, we need to remove all relations of the form $w^{\infty}=1$. 
Theorem 3.1. Let $\Gamma=\langle f, g\rangle$ be a non-elementary discrete $\mathcal{R} \mathcal{P}$ group without invariant plane. Let $\beta(f) \in(-\infty,-4)$ and let $\beta(g)=0$. Then $\gamma(f, g)=4 \cosh ^{2} u$, where $u \in \mathcal{U}, t(u) \geq 3$, and one of the following holds:

(1) If $(t(u), 2)=2$ and $\beta(f)=-4 \cosh ^{2} v / \gamma(f, g)-4$, where $v \in \mathcal{U}, t(v) \geq 3$, $(t(v), 2)=1$, then $\Gamma$ is isomorphic to $P H[\infty, t(u) / 2 ; t(v)]$.

(2) If $(t(u), 2)=2$ and $\beta(f)=-4 \cosh ^{2} v / \gamma(f, g)-4$, where $v \in \mathcal{U}, t(v) \geq 4$, $(t(v), 2)=2$, then $\Gamma$ is isomorphic to $\mathcal{S}_{2}[\infty, t(u) / 2 ; t(v) / 2]$.

(3) If $(t(u), 2)=1$ and $\beta(f)=-4 \cosh ^{2} v-4$, where $v \in \mathcal{U}, t(v) \geq 3,(t(v), 2)=$ 1 , then $\Gamma$ is isomorphic to $P[\infty, t(u) ; t(v)]$.

(4) If $(t(u), 2)=1$ and $\beta(f)=-4 \cosh ^{2} v-4$, where $v \in \mathcal{U}, t(v) \geq 4,(t(v), 2)=$ 2 , then $\Gamma$ is isomorphic to $G \operatorname{Tet}_{1}[\infty, t(u) ; t(v) / 2]$.

Proof. Suppose $(t(u), 2)=2$, that is the dihedral angle of $\mathcal{P}^{*}$ between $\sigma$ and $\tau$ is $2 \pi / m$ with $m$ even, $\infty$, or $\bar{\infty}$. Consider a polyhedron $\widetilde{\mathcal{P}}$ bounded by $\sigma, \tau$, $\sigma^{\prime}=R_{\omega}(\sigma), \tau^{\prime}=R_{\omega}(\tau), \eta$, and $\delta$. Applying the Poincaré theorem to $\widetilde{\mathcal{P}}$ and the side pairing transformations $g, g^{\prime}=R_{\sigma} R_{\omega}, e$, and $e_{f}$, one can see that $\left\langle g, g^{\prime}, e_{f}, e\right\rangle$ is isomorphic to $\widetilde{\Gamma}$ and has the presentation

$$
\left\langle f, g, e \mid g^{\infty}=e^{2}=(e f)^{2}=(e g)^{2}=\left(g f g f^{-1}\right)^{m / 2}=\left(f^{-1} g f e\right)^{p}=1\right\rangle .
$$

If $p$ is odd, then $e \in\langle f, g\rangle$ and $\widetilde{\Gamma}=\Gamma \cong P H[\infty, m / 2 ; p]$.

If $p$ is even, $\infty$, or $\bar{\infty}$, then $\widetilde{\Gamma}$ contains $\Gamma$ as a subgroup of index 2 and has presentation $\mathcal{S}_{2}[\infty, m / 2 ; p / 2]$. In order to see this, one can apply the Poincaré theorem to a polyhedron $\mathcal{P}$ bounded by $\tau, \sigma, \tau^{\prime}, \sigma^{\prime}, \eta$, and $e_{f}(\eta)$, and side-pairing transformations $f, g$, and $g^{\prime}=f g^{-1} f^{-1}$.

The proof for $(t(u), 2)=1$ is analogous. In this case we need to use the polyhedron $\mathcal{Q}^{*}$ as the starting point.

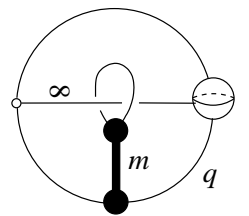

(a) $\pi_{1}^{\text {orb }}(Q) \cong P H[\infty, m ; q]$ $m \geq 2, q \geq 3$

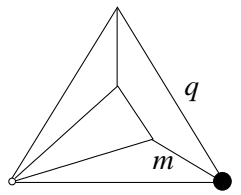

(b) $\pi_{1}(Q)^{\text {orb }} \cong P[\infty, m ; q]$ $m \geq 3, q \geq 3$

Figure 4. Orbifolds embedded in $\mathbb{S}^{3}$

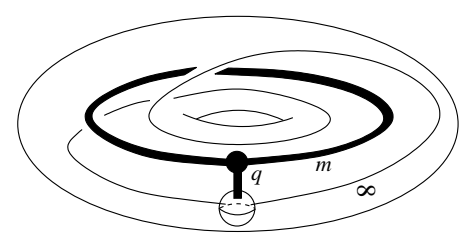

(a) $\pi_{1}^{\text {orb }}(Q) \cong \mathcal{S}_{2}[\infty, m ; q]$ $m \geq 2, q \geq 2$

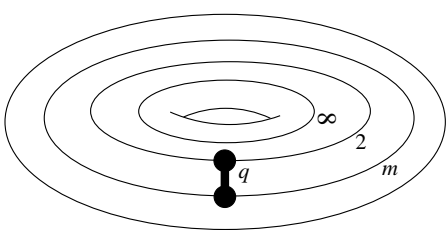

(b) $\pi_{1}^{\text {orb }}(Q) \cong G T e t_{1}[\infty, m ; q]$ $m \geq 3, q \geq 2$

Figure 5. Orbifolds embedded in Seifert fibred spaces 
The orbifolds $Q(\Gamma)$ for the groups described in Theorem 3.1 can be obtained from corresponding fundamental polyhedra. In Figures 4 and 5 we schematically draw singular sets, cusps, and boundary components of $Q(\Gamma)$ by using fat vertices and fat edges. Roughly speaking, a fat vertex is either an interior point, or is removed, or removed together with its regular neighbourhood depending on the indices. A fat edge can be labelled by $\infty$ or $\bar{\infty}$. If the index at a fat edge is $\infty$, then the egde corresponds to a cusp, and if the index is $\bar{\infty}$, the edge is removed together with its regular neighbourhood. For details, see [12].

In Figure 4 orbifolds are embedded in $\mathbb{S}^{3}$ so that $\infty$ is a non-singular interior point of $Q(\Gamma)$. Note that the volume of $Q(P H[\infty, m ; q])$ is always infinite and $Q(P[\infty, m ; q])$ is always non-compact.

Let $T(n)$ be a Seifert fibred solid torus obtained from a trivial fibred solid torus $D^{2} \times \mathbb{S}^{1}$ by cutting it along $D^{2} \times\{x\}$ for some $x \in \mathbb{S}^{1}$, rotating one of the discs through $2 \pi / n$ and glueing back together.

Denote by $\mathcal{S}(n)$ a space obtained by glueing two copies of $T(n)$ along their boundaries fibre to fibre. Clearly, $\mathcal{S}(n)$ is homeomorphic to $\mathbb{S}^{2} \times \mathbb{S}^{1}$ and is $n$-fold covered by trivially fibred $\mathbb{S}^{2} \times \mathbb{S}^{1}$. There are two critical fibres whose length is $n$ times shorter than the length of a regular fibre.

In Figure 5(a), orbifolds are embedded in Seifert fibre spaces $\mathcal{S}(2)=T(2) \cup$ $T(2)$. We draw only the solid torus that contains singular points (or boundary components). The other fibred torus is meant to be attached and is not shown. If $m<\infty$, the orbifold $Q\left(\mathcal{S}_{2}[\infty, m ; q]\right)$ is embedded in $\mathcal{S}(2)$ in such a manner that the axis of order $m$ lies on a critical fibre of $\mathcal{S}(2)$. The removed regular fibre gives rise to a cusp.

In Figure 5(b), orbifolds are embedded in trivially fibred space $\mathbb{S}^{2} \times \mathbb{S}^{1}$. The rank 2 cusp corresponds to the subgroup of $G T e t_{1}[\infty, m ; q]$ generated by $x$ and $z$.

\section{Structure of the Slice $S_{\infty}$}

Recall that

$$
S_{\infty}=\{(\gamma, \beta):(\beta, 0, \gamma) \text { are parameters for some }\langle f, g\rangle \in \mathcal{D} \mathcal{R} \mathcal{P}\},
$$

where $\mathcal{D} \mathcal{R} \mathcal{P}$ denotes the class of all non-elementary discrete $\mathcal{R} \mathcal{P}$ groups.

To investigate the slice $S_{\infty}$, we split the plane $(\gamma, \beta)$ as follows.

1. If $\beta=-4$ then by 91. Theorem 2], the group $\langle f, g\rangle$ has an invariant plane. We use [5] to find all discrete groups on the line $\beta=-4$.

2. If $\beta>-4$ and $\gamma>0$ then the group $\langle f, g\rangle$ is conjugate to a subgroup of $\operatorname{PSL}(2, \mathbb{R})$. More precisely, if $-4<\beta<0$ then $f$ is elliptic and the axis of $f$ is orthogonal to an invariant plane of $g$ and if $\beta=0$ then the fixed points of $f$ and $g$ lie in their common invariant plane. Discreteness criteria in terms of traces of $f, g$, and $f g$ were given in [14]. For $\beta>0$, an algorithm to decide whether $f$ and $g$ generate a discrete group was given in [8].

3. If $\beta>-4$ and $\gamma<0$ then $f$ is elliptic, parabolic, or hyperbolic and the group $\langle f, g\rangle$ is known to be truly spatial. Discrete such groups are described in 11], where $\beta$ and $\gamma$ are found explicitly.

4. If $\beta<-4$ and $\gamma<0$ then $f$ is $\pi$-loxodromic whose axes lies in an invariant plane of $g$. Then this plane is invariant under action of $\langle f, g\rangle$ and $f$ acts as 
a glide-reflection on it. A geometrical description of such discrete groups was given in 13.

5. The case of $\beta<-4$ and $\gamma>0$ was treated in Section 2 of the present paper.

We will obtain explicit formulas for $\beta$ and $\gamma$ in the cases 2 and 4 above and completely describe the structure of the slice $S_{\infty}$. We will pay special attention to the subsets of $S_{\infty}$ corresponding to free groups.

First, we need the following elementary facts.

Lemma 4.1. If $f, g \in \operatorname{PSL}(2, \mathbb{C})$ and $g$ is parabolic, then

$$
\gamma(f, g)=(\operatorname{tr}(f g)-\operatorname{sign}(\operatorname{tr} g) \cdot \operatorname{tr} f)^{2} .
$$

Proof. By the Fricke identity, we have

$$
\begin{aligned}
\gamma(f, g) & =\operatorname{tr}[f, g]-2 \\
& =\operatorname{tr}^{2} f+\operatorname{tr}^{2} g+\operatorname{tr}^{2}(f g)-\operatorname{tr} f \cdot \operatorname{tr} g \cdot \operatorname{tr}(f g)-4 \\
& =(\operatorname{tr}(f g)-\operatorname{sign}(\operatorname{tr} g) \cdot \operatorname{tr} f)^{2},
\end{aligned}
$$

since $\operatorname{tr}^{2} g=4$.

Lemma 4.2. If $f, g \in \operatorname{PSL}(2, \mathbb{C})$ and $\operatorname{tr} g=2$, then

$$
\operatorname{tr}\left(f g^{k}\right)=k(\operatorname{tr}(f g)-\operatorname{tr} f)+\operatorname{tr} f .
$$

Proof. By substituting $\operatorname{tr} g=2$ into the recurrent formula

$$
\operatorname{tr}\left(f g^{k}\right)=\operatorname{tr}\left(f g^{k-1}\right) \operatorname{tr} g-\operatorname{tr}\left(f g^{k-2}\right),
$$

we immediately get the result.

Remark 4.3. Suppose that $f$ is non-primitive elliptic of finite order $n$, i.e., $\beta(f)=$ $-4 \sin ^{2}(q \pi / n)$, where $(q, n)=1,1<q<n / 2$. Then there exists an integer $r$ so that $f^{r}$ is primitive of the same order. Obviously, $\langle f, g\rangle=\left\langle f^{r}, g\right\rangle$ and $\beta\left(f^{r}\right)=$ $-4 \sin ^{2}(\pi / n)$. By [7], $\gamma\left(f^{r}, g\right)=\left(\beta\left(f^{r}\right) / \beta(f)\right) \gamma(f, g)$.

It is natural to introduce the constant

$$
C(q, n)=\frac{\sin ^{2}(q \pi / n)}{\sin ^{2}(\pi / n)}=\frac{\beta(f)}{\beta\left(f^{r}\right)} \geq 1
$$

that plays an important role in parameters calculation concerning groups with elliptic elements. It is also convenient to consider a parabolic element $f$ as a limit rotation of order $n=\infty$ and write $0=\beta(f)=-4 \sin ^{2}(\pi / n)$ with $C(q, n)=$ $C(1, n)=1$.

4.1. $-4 \leq \beta \leq 0$. This means that $f$ is either elliptic or parabolic. Obviously, if $f$ is elliptic of infinite order, then $\langle f, g\rangle$ is not discrete. So we assume that $\beta=-4 \sin ^{2}(q \pi / n)$, where $(q, n)=1$ and $1 \leq q<n / 2$, including $\beta=0$.

Theorem 4.4. Let $\Gamma=\langle f, g\rangle \subset \operatorname{PSL}(2, \mathbb{C})$ have parameters $(\beta, 0, \gamma)$ with $\gamma \in$ $\mathbb{R} \backslash\{0\}$. Let $\beta=-4 \sin ^{2}(q \pi / n)$, where $(q, n)=1$ and $1 \leq q<n / 2$, including $\beta=0$. Then $\Gamma$ is discrete if and only if one of the following holds:

(1) $\gamma=-4 C(q, n) \cosh ^{2} u$, where $u \in \mathcal{U}$ and $t(u) \geq 3$;

(2) $\gamma=4 C(q, n)(\cos (\pi / n)+\cosh u)^{2}$, where $u \in \mathcal{U}$;

(3) $\beta=0$ and $\gamma=4(1+\cos (2 \pi / k))^{2}$, where $k \geq 3$ is odd. 
Proof. Let us prove the theorem for $q=1$; in order to get the result for $q>1$, we only need to apply Remark 4.3

If $n=2$ then $\beta=-4$ and, by [5. Theorem 4.15], $\Gamma$ is discrete if and only if $\gamma= \pm 4 \cosh ^{2} u$, where $u \in \mathcal{U}$ with $t(u) \geq 3$.

If $2<n \leq \infty$ and $\gamma<0$, then, by [11, Corollary 2.5], $\Gamma$ is discrete if and only if $\gamma=-4 \cosh ^{2} u$, where $u \in \mathcal{U}$ and $t(u) \geq 3$.

Assume that $2<n<\infty$ and $\gamma>0$. In this case $\Gamma$ is conjugate to a subgroup of $\operatorname{PSL}(2, \mathbb{R})$ and we can apply Knapp's results 14 to compute $\gamma$. Conjugate $\Gamma$ so that $\infty$ is the fixed point of $g$. By replacing, if necessary, $f$ with $f^{-1}$ and $g$ with $g^{-1}$, we may assume that

$$
f=\left(\begin{array}{ll}
a & b \\
c & d
\end{array}\right) \quad \text { and } g=\left(\begin{array}{rr}
-1 & \tau \\
0 & -1
\end{array}\right)
$$

where $a d-b c=1, a+d=-2 \cos (\pi / n)$ with $n \in \mathbb{Z}, b>0$, and $\tau>0$.

One can show that $\operatorname{tr}(f g)<2$. By [14, Proposition 4.1], $\Gamma$ is discrete if and only if $\operatorname{tr}(f g) \leq-2$ or $\operatorname{tr}(f g)=-2 \cos (\pi / k)$, where $k \geq 2$ is an integer, that is $\operatorname{tr}(f g)=-2 \cosh u$, where $u \in \mathcal{U}$. Hence, by Lemma 4.1 $\gamma=(\operatorname{tr}(f g)+\operatorname{tr} f)^{2}=$ $(2 \cosh u+2 \cos (\pi / n))^{2}$.

So it remains to consider the case when $n=\infty$ (i.e., $\beta=0$ ) and $\gamma>0$. Again, we normalize $\Gamma$ so that $g$ is as above and $f=\left(\begin{array}{rr}-1 & 0 \\ -1 & -1\end{array}\right)$. By [14, Proposition 4.2], such a group is discrete if and only if $\tau \geq 4$ or $\tau=2+2 \cos (2 \pi / k)$ for an integer $k \geq 3$. Since in this case $\gamma=\tau^{2}$, we have that $\gamma \geq 16$ or $\gamma=(2+2 \cos (2 \pi / k))^{2}$, which can be written as $\gamma=4(1+\cosh u)^{2}$, where $u \in \mathcal{U}$, or $\gamma=4(1+\cos (2 \pi / k))^{2}$ for odd $k \geq 3$.

Remark 4.5. If $-4 \leq \beta \leq 0$ then $\Gamma$ is discrete and free if and only if $\beta=0$ and $\gamma \in(-\infty,-4] \cup[16,+\infty)$.

The parameters from the infinite strip $-4 \leq \beta \leq 0$ are displayed in Figure 6 If $\beta=-4 \sin ^{2}(q \pi / n)$ is fixed, then there exist values $\gamma_{1}(\beta)<0$ and $\gamma_{2}(\beta)>0$ so that $\Gamma$ is discrete in the union of two rays $\left(-\infty, \gamma_{1}(\beta)\right] \cup\left[\gamma_{2}(\beta),+\infty\right)$. There are only countably many discrete groups in $\left(\gamma_{1}(\beta), \gamma_{2}(\beta)\right)$ with accumulation points $\gamma_{1}(\beta)$ and $\gamma_{2}(\beta)$.

Moreover, if we denote $\beta_{n}^{q}=-4 \sin ^{2}(q \pi / n)$, then

$$
\gamma_{1}\left(\beta_{n}^{q}\right)<\gamma_{1}\left(\beta_{n}^{1}\right)<\gamma_{2}\left(\beta_{n}^{1}\right)<\gamma_{2}\left(\beta_{n}^{q}\right) \text { for all } 1<q<n / 2 .
$$

4.2. $\beta>0$. In this case $f$ is hyperbolic.

Theorem 4.6 (11, Corollary 2.5]). Let $\Gamma=\langle f, g\rangle \subset \operatorname{PSL}(2, \mathbb{C})$ have parameters $(\beta, 0, \gamma)$ with $\beta>0$ and $\gamma<0$. Then $\Gamma$ is discrete if and only if $\gamma=-4 \cosh ^{2} u$, where $u \in \mathcal{U}, t(u) \geq 3$.

Remark 4.7. From 11, $\Gamma$ with parameters $(\beta, 0, \gamma)$, where $\beta \geq 0$ and $\gamma<0$ is free if and only if $(\gamma, \beta)$ lies in the region

$$
A=\{(\gamma, \beta): \gamma \leq-4, \beta \geq 0\} .
$$

Theorem 4.8. Let $\Gamma=\langle f, g\rangle \subset \operatorname{PSL}(2, \mathbb{C})$ have parameters $(\beta, 0, \gamma)$ with $\beta>0$ and $\gamma>0$. Let $k=\left\lceil\frac{\sqrt{\beta+4}-2}{\sqrt{\gamma}}\right\rceil$. The group $\Gamma$ is discrete if and only if one of the following holds: 

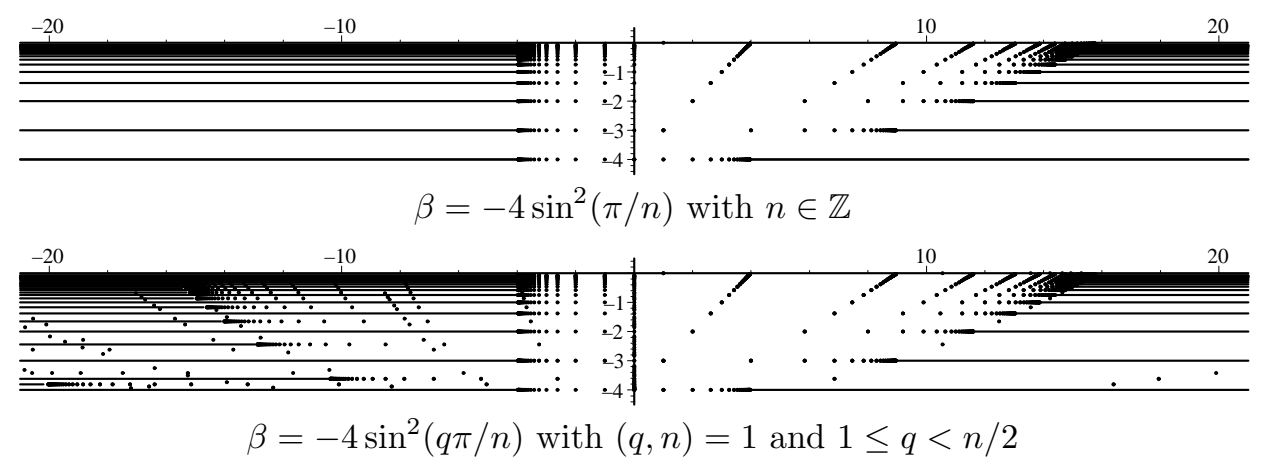

FIgURE 6. Structure of the strip $-4 \leq \beta \leq 0$

(1) $\beta=(k \sqrt{\gamma}+2)^{2}-4$ and $\gamma=16 \cosh ^{4} u$, where $u \in \mathcal{U}$ and $t(u) \geq 3$;

(2) $\beta=(k \sqrt{\gamma} \pm 2 \cos (q \pi / n))^{2}-4$ and $\gamma=4 C(q, n)(\cos (\pi / n)+\cosh u)^{2}$, where $(q, n)=1,1 \leq q<n / 2$, and $u \in \mathcal{U}$;

(3) $\beta=(k \sqrt{\gamma}-2 \cosh u)^{2}-4$ and $\gamma>4(1+\cosh u)^{2}$, where $u \geq 0$.

Proof. Since $\gamma>0$, the axis of $f$ lies in an invariant plane of $g$, so $\Gamma=\langle f, g\rangle$ is conjugate to a subgroup of $\operatorname{PSL}(2, \mathbb{R})$. In 8 , an algorithm for determining whether such a group is discrete was given. We will apply this algorithm and calculate parameters for each discrete group.

Normalize $\Gamma$ so that $\infty$ is the fixed point of $g$ and \pm 1 are the fixed points of $f$. Then we can write

$$
f=\left(\begin{array}{ll}
a & b \\
b & a
\end{array}\right) \text { and } g=\left(\begin{array}{ll}
1 & \tau \\
0 & 1
\end{array}\right), \text { where } a^{2}-b^{2}=1, a>1, b, \tau \in \mathbb{R} .
$$

By replacing $f$ with $f^{-1}$ and $g$ with $g^{-1}$, we may assume that $b<0$ and $\tau>0$.

Let $k$ be a positive integer such that $\operatorname{tr}\left(f g^{k}\right) \leq 2$ and $\operatorname{tr}\left(f g^{\ell}\right)>2$ for all $\ell$ with $0 \leq \ell<k$.

By Lemmas 4.1 and 4.2 we have that $k^{2} \gamma=k^{2}(\operatorname{tr}(f g)-\operatorname{tr} f)^{2}=\left(\operatorname{tr}\left(f g^{k}\right)-\operatorname{tr} f\right)^{2}$. Since $\operatorname{tr}\left(f g^{k}\right) \leq 2$ and $\operatorname{tr} f>2$,

$$
\operatorname{tr} f=k \sqrt{\gamma}+\operatorname{tr}\left(f g^{k}\right)
$$

We distinguish three cases:

1. $\operatorname{tr}\left(f g^{k}\right)=2$, that is $f g^{k}$ is parabolic. From (4.7),

$$
\beta=(k \sqrt{\gamma}+2)^{2}-4
$$

By Theorem 4.4 $\left\langle f g^{k}, g\right\rangle$ and, hence, $\langle f, g\rangle$ is discrete if and only if

$$
\begin{aligned}
& \gamma=\gamma\left(f g^{k}, g\right)=4(1+\cosh v)^{2}, \text { where } v \in \mathcal{U}, \text { or } \\
& \gamma=4(1+\cos (2 \pi / k))^{2}, \text { where } k \geq 3 \text { is odd. }
\end{aligned}
$$

These expressions can be rearranged and combined as $\gamma=16 \cosh ^{4} u$, where $u \in \mathcal{U}$ and $t(u) \geq 3$.

2. $-2<\operatorname{tr}\left(f g^{k}\right)<2$, that is $f g^{k}$ is elliptic and $\operatorname{tr}\left(f g^{k}\right)= \pm 2 \cos (q \pi / n)$, where $(q, n)=1$ and $1 \leq q<n / 2$. Hence, from (4.7),

$$
\beta=(k \sqrt{\gamma} \pm 2 \cos (q \pi / n))^{2}-4
$$

By Theorem $4.4\left\langle f g^{k}, g\right\rangle$ and, hence, $\langle f, g\rangle$ is discrete if and only if

$$
\gamma=4 C(q, n)(\cos (\pi / n)+\cosh u)^{2}, \quad \text { where } u \in \mathcal{U} .
$$


3. $\operatorname{tr}\left(f g^{k}\right) \leq-2$, that is $f g^{k}$ is hyperbolic or parabolic so we can write $\operatorname{tr}\left(f g^{k}\right)=$ $-2 \cosh u$, where $u \geq 0$. Then

$$
\beta=(k \sqrt{\gamma}-2 \cosh u)^{2}-4 .
$$

Consider the group $\left\langle g^{k-1} f, g\right\rangle$. The element $g^{k-1} f$ is hyperbolic with $\operatorname{tr}\left(g^{k-1} f\right)>2$. Therefore, one can normalize $\left\langle g^{k-1} f, g\right\rangle$ so that the attracting and repelling fixed points of $g^{k-1} f$ are $x_{a}$ and $x_{r}$, respectively, and $x_{a}<x_{r}$. Since $\operatorname{tr}\left(g^{k} f\right) \leq-2$, such a group is dicrete and free by [8, Case II]. So by Lemma 4.1] we have that

$$
\begin{aligned}
\gamma=\gamma\left(f g^{k-1}, g\right) & =\left(\operatorname{tr}\left(f g^{k}\right)-\operatorname{tr}\left(f g^{k-1}\right)\right)^{2} \\
& =(2 \cosh u+2 \cosh v)^{2}
\end{aligned}
$$

where $v$ is any positive real number.

It remains to compute $k$. Since $\operatorname{tr}\left(f g^{k}\right)=2 a+b \tau k \leq 2$, we have that $k \geq$ $(-2 a+2) /(b \tau)$. Computing $\gamma=b^{2} \tau^{2}$, we get $b \tau=-\sqrt{\gamma}$. So $k=\left\lceil\frac{\sqrt{\beta+4}-2}{\sqrt{\gamma}}\right\rceil$.

It follows from $[8$ that $\Gamma$ is free if and only if $(\gamma, \beta)$ lies in one of the regions

$$
C_{k}=\left\{(\gamma, \beta): \gamma \geq 16,((k-1) \sqrt{\gamma}+2)^{2} \leq \beta+4 \leq(k \sqrt{\gamma}-2)^{2}\right\}, k=1,2,3 \ldots
$$

4.3. $\beta<-4$. First, consider $\gamma<0$. In this case the axis of the $\pi$-loxodromic generator $f$ lies in an invariant plane of $g[9$, so $\langle f, g\rangle$ keeps this plane invariant.

Theorem 4.9. Let $\Gamma=\langle f, g\rangle \subset \operatorname{PSL}(2, \mathbb{C})$ have parameters $(\beta, 0, \gamma)$ with $\beta<-4$ and $\gamma<0$. Let $k=\left\lceil\frac{\sqrt{-\beta-4}}{\sqrt{-\gamma}}\right\rceil$. Then the group $\langle f, g\rangle$ is discrete if and only if one of the following holds:

(1) $-4(\beta+4)=((2 k-1) \sqrt{-\gamma} \pm \sqrt{-\gamma-8(1+\cosh u)})^{2}$, where $u \in \mathcal{U}$;

(2) $4(\beta+4)=(2 k-1)^{2} \gamma$ and $\gamma=-16 \cos ^{2}(\pi / p)$, where $p \geq 3$ is odd;

(3) $\beta=k^{2} \gamma-4$ and $\gamma=-4 \cosh ^{2} u$, where $u \in \mathcal{U}$ and $t(u) \geq 3$.

Proof. Let $\delta=\{(z, t): \operatorname{Im} z=0\}$ be the invariant plane of $\Gamma$. Since the axis of $f$ lies in $\delta$, we can normalize $\Gamma$ so that the fixed point of $g$ is $\infty$, the fixed points of $f$ are \pm 1 , and

$$
f=\left(\begin{array}{cc}
a i & b i \\
b i & a i
\end{array}\right), \quad g=\left(\begin{array}{cc}
1 & \tau \\
0 & 1
\end{array}\right), \quad \text { where } b^{2}-a^{2}=1, a>1, b, \tau \in \mathbb{R} .
$$

Further, replacing $f$ with $f^{-1}$ and $g$ with $g^{-1}$, we can assume that $b<0$ and $\tau>0$. Since $b$ is negative, +1 is the repelling fixed point of $f$ and -1 is attracting.

Let $e$ be the half-turn whose axis passes through the fixed point of $g$ orthogonally to the axis of $f$. That is $e$ fixes 0 and $\infty$. Let $e_{f}$ and $e_{1}$ be half-turns such that $f=e e_{f}$ and $g=e_{1} e$. Since $f$ is $\pi$-loxodromic, the axis of $e_{f}$ intersects the axis of $f$ (and the plane $\delta$ ) orthogonally; denote the intersection point by $A$. Further, since $g$ is parabolic and keeps $\delta$ invariant, the axis of $e_{1}$ fixes $\infty$ and lies in the plane $\delta$. It is easy to calculate that

$$
e=\left(\begin{array}{rr}
i & 0 \\
0 & -i
\end{array}\right), \quad e_{f}=\left(\begin{array}{rr}
a & b \\
-b & -a
\end{array}\right), \quad e_{1}=\left(\begin{array}{cc}
i & \tau \\
0 & -i
\end{array}\right) .
$$

Consider half-turns $e_{k-1}=g^{k-1} e$ and $e_{k}=g^{k} e$ such that $A$ lies in the region bounded by the axes of $e_{k-1}$ and $e_{k}$ in the plane $\delta$, see Figure 7 It is easy to 
calculate that $A=-a / b-j / b$. Since $e_{k}$ fixes $\infty$ and $\tau k / 2$, we have that

$$
A \in\left\{(z, t): \frac{\tau(k-1)}{2}<\operatorname{Re} z \leq \frac{\tau k}{2}, \operatorname{Im} z=0, t>0\right\} .
$$

Hence, we can immediately determine $k$.

$$
\frac{\tau(k-1)}{2}<-\frac{a}{b} \leq \frac{\tau k}{2} .
$$

Therefore, since $2 a=-i \operatorname{tr} f=\sqrt{-\beta-4}$ and $b \tau=-\sqrt{-\gamma}$,

$$
k=\left\lceil-\frac{2 a}{b \tau}\right\rceil=\left\lceil\frac{\sqrt{-\beta-4}}{\sqrt{-\gamma}}\right\rceil .
$$

It is easy to see that $\Gamma$ is discrete if and only if $\widetilde{\Gamma}=\left\langle e_{f}, e_{k-1}, e_{k}\right\rangle$ is. Following [13, we give geometric conditions for $\widetilde{\Gamma}$ to be discrete.

Suppose that $A \notin \operatorname{axis}\left(e_{k}\right)$; see Figure [7(a). By [13], $\widetilde{\Gamma}$ is discrete if either

(a) the angle $\phi$ between $e_{k-1}$ and $e_{f}\left(e_{k}\right)$ is of the form $\pi / p$, where $p \geq 2$ is an integer, $\infty$, or $\bar{\infty}$; or

(b) $\phi=2 \pi / p$, where $p \geq 3$ is odd and the bisector of $\phi$ passes through $A$.

Suppose that $A \in \operatorname{axis}\left(e_{k}\right)$; see Figure [(b). By [13, $\widetilde{\Gamma}$ is discrete if

(c) the angle $\psi$ made by axis $\left(e_{k-1}\right)$ and $\operatorname{axis}\left(\tilde{e}_{f}\right)$ is of the form $\pi / p, p \geq 3$ is an integer, $\infty$, or $\bar{\infty}$, where $\tilde{e}_{f}=e_{k} e_{f}$ is the half-turn whose axis passes through $A$ orthogonally to $\operatorname{axis}\left(e_{k}\right)$ in the plane $\delta$.

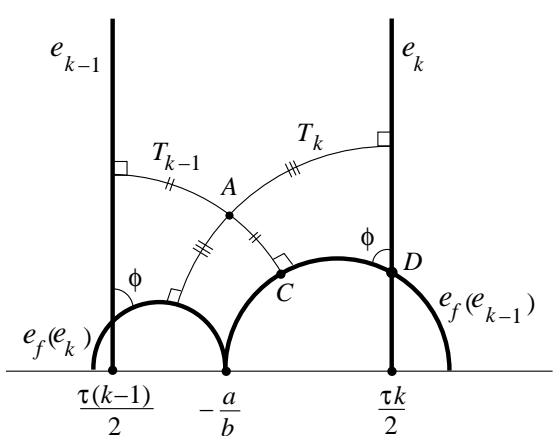

(a)

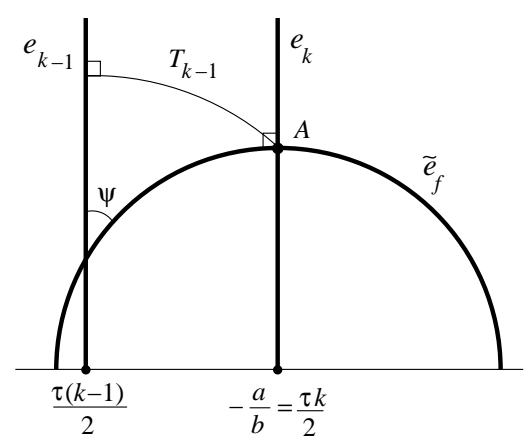

(b)

Figure 7. The invariant plane $\delta$

There are no other discrete groups. So, we need to calculate the parameters $\beta$ and $\gamma$ in each of the cases (a), (b), and (c).

Assume that we are in case (a) or (b). Then each $g^{\ell} f=e_{\ell} e_{f}, \ell \in \mathbb{Z}$, is a $\pi$ loxodromic element with translation length $2 T_{\ell}$ and $\operatorname{tr}\left(g^{\ell} f\right)= \pm 2 i \sinh T_{\ell}$, where $T_{\ell}$ is the distance between $e_{\ell}$ and $A$. Moreover, from the matrix representation, $\operatorname{tr}\left(g^{\ell} f\right)=2 a i+b \tau \ell i$. The inequalities (4.8) enable us to determine the signs of $\operatorname{tr}\left(f g^{k-1}\right)$ and $\operatorname{tr}\left(f g^{k}\right)$ :

$$
\operatorname{tr}\left(f g^{k}\right)=-2 i \sinh T_{k} \quad \text { and } \quad \operatorname{tr}\left(f g^{k-1}\right)=+2 i \sinh T_{k-1} .
$$


Suppose that $p<\infty$. Simple calculations in the plane $\delta$ show that

$$
\sinh C D=\frac{1+\cos \phi \cosh \left(2 T_{k-1}\right)}{\sin \phi \sinh \left(2 T_{k-1}\right)}
$$

and, on the other hand,

$$
\sinh C D=\frac{\sinh T_{k}+\cos \phi \sinh T_{k-1}}{\sin \phi \cosh T_{k-1}}
$$

So, we obtain

$$
2(1+\cos \phi)=4 \sinh T_{k-1} \sinh T_{k}=\operatorname{tr}\left(f g^{k-1}\right) \operatorname{tr}\left(f g^{k}\right) .
$$

Applying Lemmas 4.1 and 4.2 and the facts that $\operatorname{tr} f=i \sqrt{-\beta-4}$ and $\operatorname{tr}(f g)-\operatorname{tr} f=$ $b \tau i=-i \sqrt{-\gamma}$, we get

$$
\begin{aligned}
2(1+\cos \phi) & =[(k-1)(\operatorname{tr}(f g)-\operatorname{tr} f)+\operatorname{tr} f] \cdot[k(\operatorname{tr}(f g)-\operatorname{tr} f)+\operatorname{tr} f] \\
& =k(k-1)(\operatorname{tr}(f g)-\operatorname{tr} f)^{2}+(2 k-1) \cdot \operatorname{tr} f \cdot(\operatorname{tr}(f g)-\operatorname{tr} f)+\operatorname{tr}^{2} f \\
& =k(k-1) \gamma+(2 k-1) \sqrt{-\beta-4} \sqrt{-\gamma}+\beta+4 .
\end{aligned}
$$

Hence, $-4(\beta+4)=((2 k-1) \sqrt{-\gamma} \pm \sqrt{-8(1+\cos \phi)-\gamma})^{2}$, where $\phi=\pi / p, p \geq 2$ is an integer. Analogous calculation can be done for $p=\infty$ and $p=\bar{\infty}$, and we obtain item (1) of the theorem.

In case (b), in addition, $T_{k-1}=T_{k}$. Then $\operatorname{tr}\left(f g^{k}\right)=-\operatorname{tr}\left(f g^{k-1}\right)$ and by Lemmas 4.1 and 4.2 we have

$$
2 \sqrt{-\beta-4}=(2 k-1) \sqrt{-\gamma}
$$

Therefore, $2(1+\cos \phi)=-\operatorname{tr}^{2}\left(f g^{k}\right)=(-k \sqrt{-\gamma}+\sqrt{-\beta-4})^{2}=-\gamma / 4$. Hence, since $\phi=2 \pi / p, \gamma=-16 \cos ^{2}(\pi / p)$.

Now assume that we are in case (c) and $p<\infty$. Since in this case $e_{k} e_{f}=\tilde{e}_{f}$ is an ellitic element of order $2, \operatorname{tr}\left(g^{k} f\right)=0$. Therefore, since $\operatorname{tr}\left(g^{k} f\right)=-k i \sqrt{-\gamma}+$ $i \sqrt{-\beta-4}$, we have that $\beta=k^{2} \gamma-4$.

Further, since $\operatorname{tr}\left(f g^{k-1}\right)=2 i \sinh T_{k-1}$ and, from the plane $\delta, \sinh T_{k-1}=\cos \psi$, we have that

$$
\begin{aligned}
4 \cos ^{2} \psi=4 \sinh ^{2} T_{k-1} & =-((k-1)(\operatorname{tr}(f g)-\operatorname{tr} f)+\operatorname{tr} f)^{2} \\
& =(-(k-1) \sqrt{-\gamma}+\sqrt{-\beta-4})^{2} \\
& =(-(k-1) \sqrt{-\gamma}+k \sqrt{-\gamma})^{2} \\
& =-\gamma .
\end{aligned}
$$

Thus, $\gamma=-4 \cos ^{2}(\pi / p)$, where $p \geq 3$ is an integer. Analogous calculations can be done for $p=\infty$ and $p=\bar{\infty}$ and we obtain item (3) of the theorem.

Remark 4.10. If $\beta<-4$ and $\gamma<0$, then $\langle f, g\rangle$ is free if and only if $(\gamma, \beta)$ lies in one of the regions $D_{k}, k=1,2,3, \ldots$, given by

$$
\begin{aligned}
D_{k}=\{ & (\gamma, \beta): \gamma \leq-16, \\
& \left.\frac{((2 k-1) \sqrt{-\gamma}-\sqrt{-\gamma-16})^{2}}{-4} \geq \beta+4 \geq \frac{((2 k-1) \sqrt{-\gamma}+\sqrt{-\gamma-16})^{2}}{-4}\right\} .
\end{aligned}
$$

When $\gamma>0$, the parameters were described in Theorem 2.6 Here we just note that for $\gamma>0$ and $\beta<0$, the group $\langle f, g\rangle$ is free if and only if $(\gamma, \beta)$ lies in the region

$$
B=\{(\gamma, \beta): \gamma \geq 4, \beta+4 \leq-4 / \gamma\}
$$




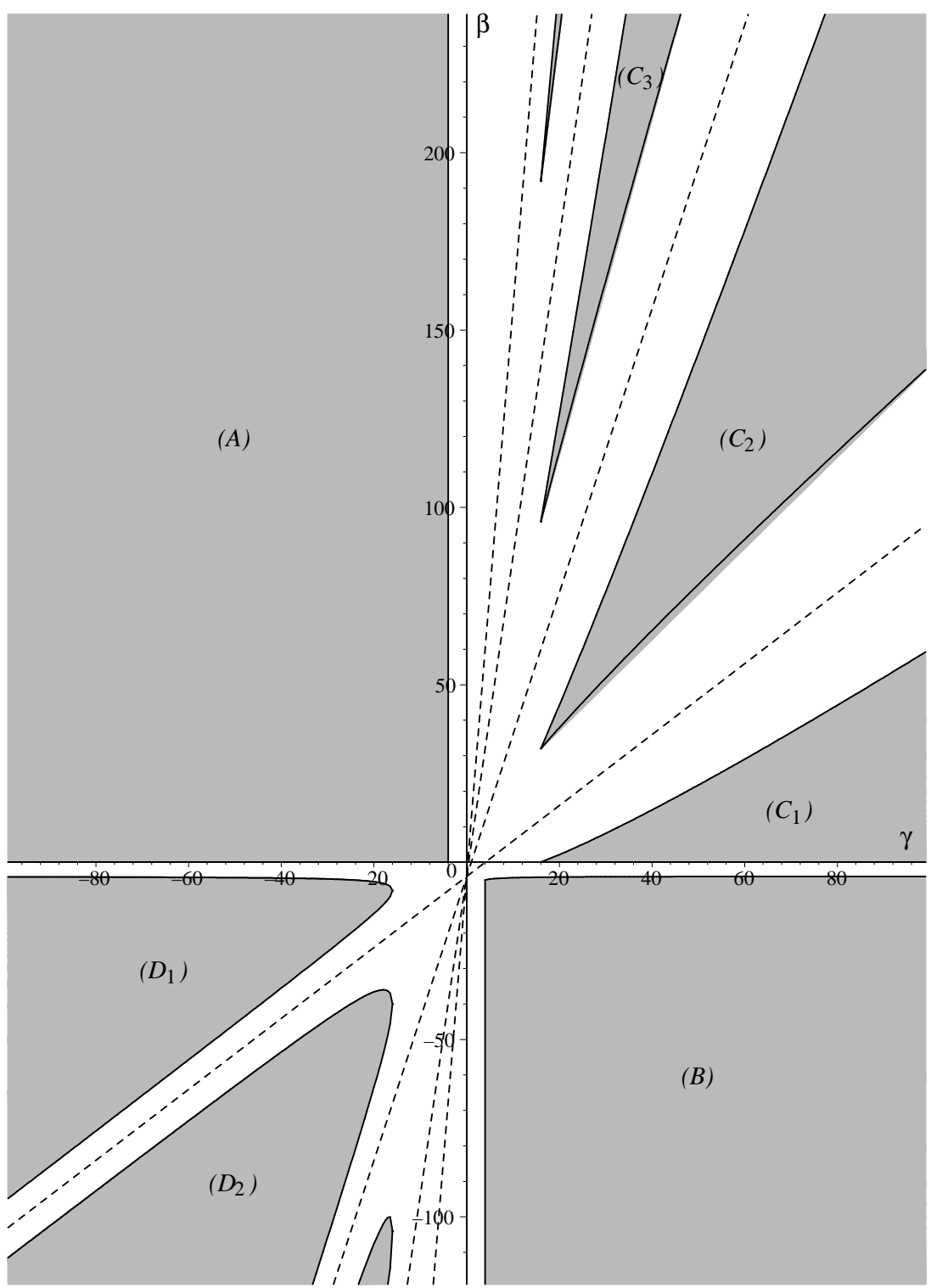

$$
\begin{aligned}
A= & \{(\gamma, \beta): \gamma \leq-4, \beta \geq 0\} \\
B= & \{(\gamma, \beta): \gamma \geq 4, \beta+4 \leq-4 / \gamma\} \\
C_{k}= & \left\{(\gamma, \beta): \gamma \geq 16,((k-1) \sqrt{\gamma}+2)^{2} \leq \beta+4 \leq(k \sqrt{\gamma}-2)^{2}\right\} \\
D_{k}= & \{(\gamma, \beta): \gamma \leq-16, \\
& \left.\frac{((2 k-1) \sqrt{-\gamma}+\sqrt{-\gamma-16})^{2}}{-4} \leq \beta+4 \leq \frac{((2 k-1) \sqrt{-\gamma}-\sqrt{-\gamma-16})^{2}}{-4}\right\}
\end{aligned}
$$

Dashed lines $\beta=k^{2} \gamma-4, k=1,2,3, \ldots$

FiguRE 8 . The discrete free groups 
Finally, we are able to draw those subsets of $S_{\infty}$ that correspond to discrete free groups. These subsets are shown in Figure 8. The dashed lines $\beta=k^{2} \gamma-4$ are plotted to show a certain symmetry of $S_{\infty}$.

The other discrete groups contain elliptic elements. Their parameters are represented by lines, parabolas, hyperbolas, and points accumulating, as orders of elliptic elements tend to $\infty$, to the regions of free groups.

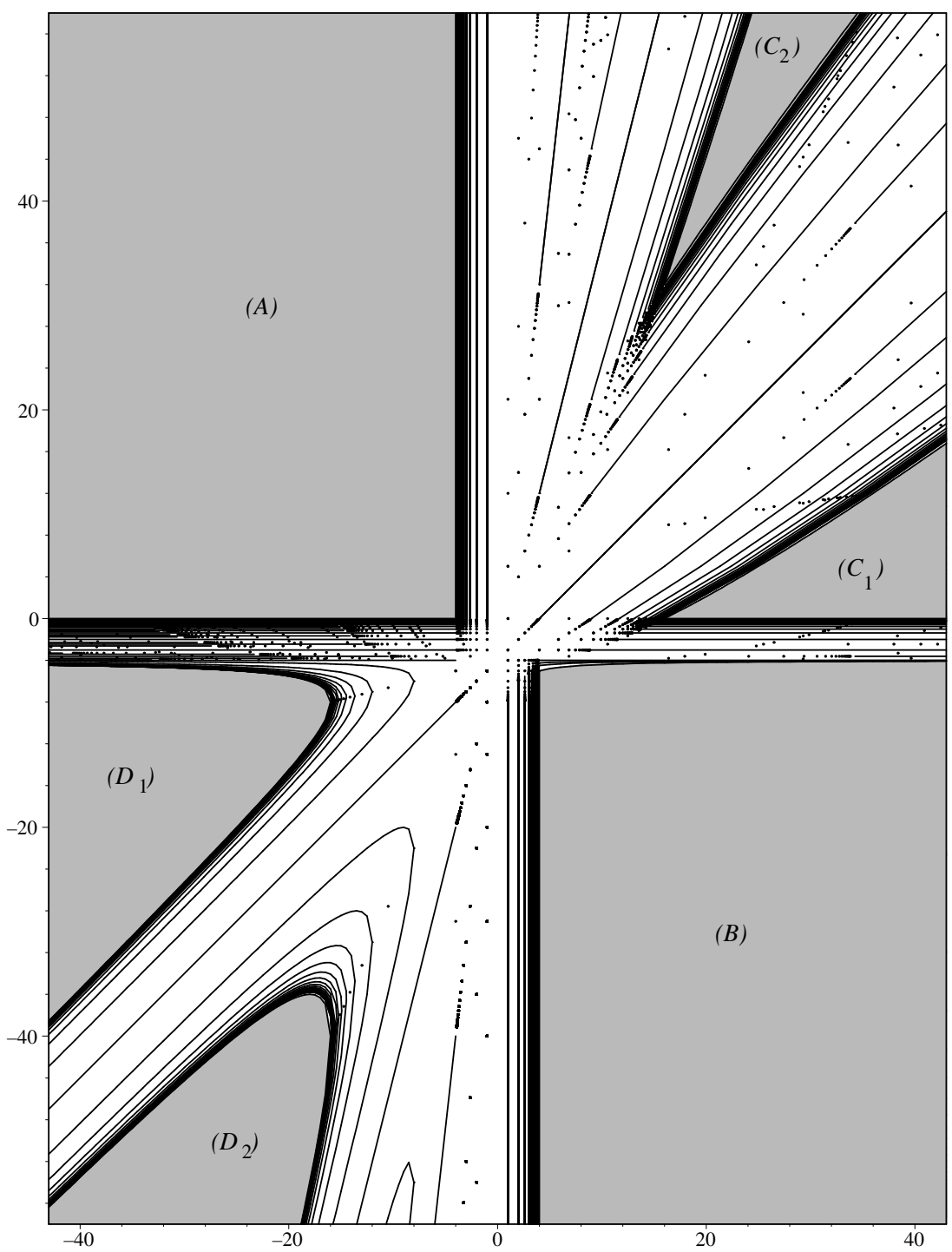

Figure 9 . The structure of the slice $S_{\infty}$

In Figure 9 the whole picture for the slice $S_{\infty}$ is shown to give an idea of the structure of $S_{\infty}$. The formulas for $\beta$ and $\gamma$ obtained in Theorems 2.6 4.4 4.6 4.8. and 4.9] were programmed with the package Maple 7.0 for some (sufficiently large) values of independent variables like $n, q \in \mathbb{Z}$ and $u, v \in \mathcal{U}$ and plotted on the plane $(\gamma, \beta)$. 
The most interesting families of parameters appear when $\gamma$ and $\beta$ are of the same sign. For a fixed $k$, the hyperbolas

$$
-4(\beta+4)=((2 k-1) \sqrt{-\gamma} \pm \sqrt{-\gamma-8(1+\cos (\pi / p))})^{2},
$$

where $p \geq 2$ is an integer, form a one-parameter family of curves converging to the boundary of $D_{k}$ as $p \rightarrow \infty$. Each hyperbola has the asymptotes $\beta=(k-1)^{2} \gamma-$ $4 k(1+\cos (\pi / p))+4$ and $\beta=k^{2} \gamma+4 k(1+\cos (\pi / p))-4$, which are obviously parallel to $\beta=(k-1)^{2} \gamma-4$ and $\beta=k^{2} \gamma-4$, respectively.

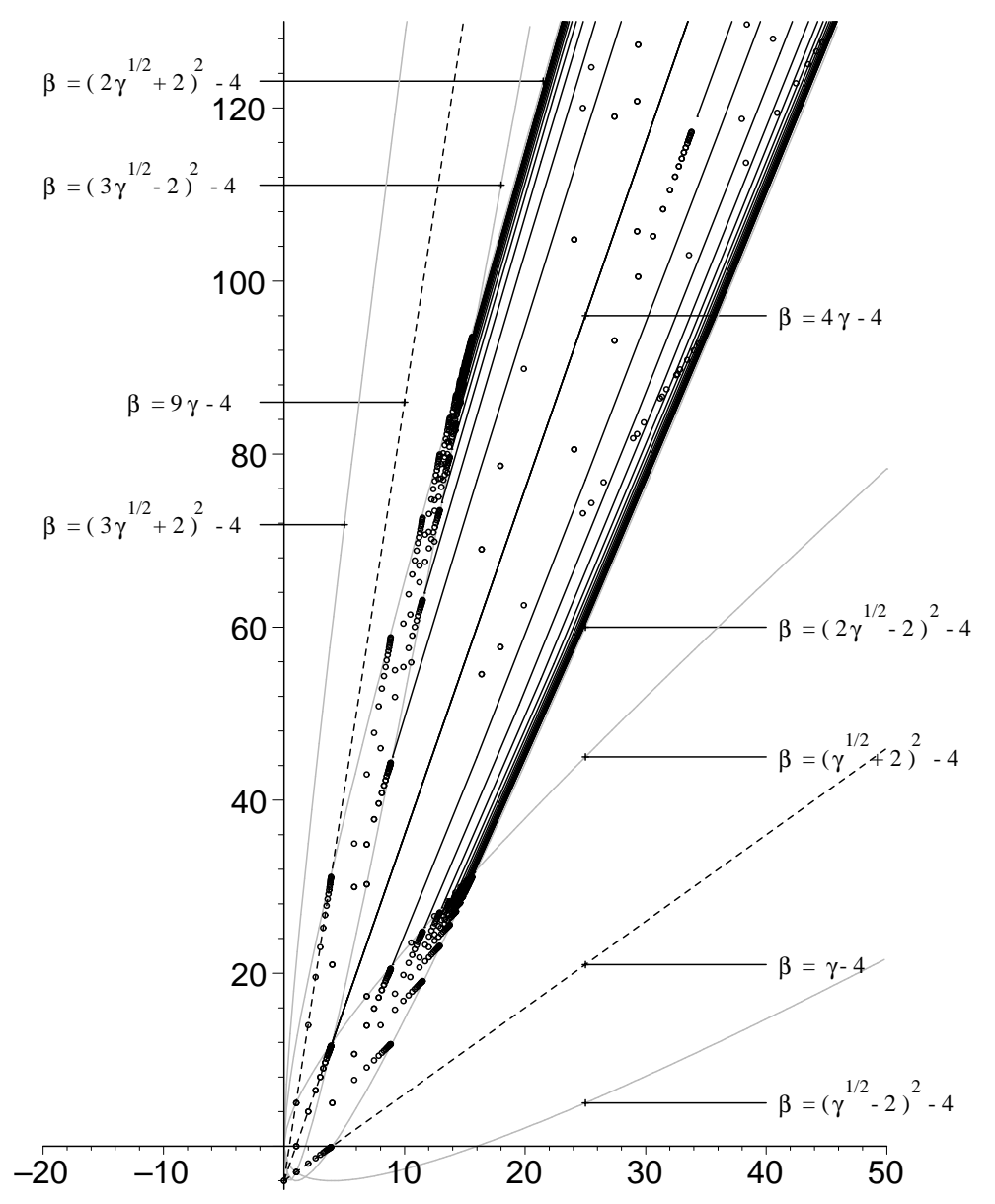

FiguRE 10. The structure of $\Sigma_{2}$

For $\gamma>0$ and $\beta>0$, consider a one-parameter family of parabolas $\beta_{k}=$ $(k \sqrt{\gamma} \pm 2)^{2}-4$. Let $\Sigma_{k}$ be the domain bounded by $\beta_{k}$ :

$$
\Sigma_{k}=\left\{(\gamma, \beta):(k \sqrt{\gamma}-2)^{2} \leq \beta+4 \leq(k \sqrt{\gamma}+2)^{2}\right\} .
$$

Within each $\Sigma_{k}$, the parameters for discrete groups are given by

$$
\left\{\begin{array}{l}
\beta=(k \sqrt{\gamma} \pm 2 \cos (q \pi / n))^{2}-4 \\
\gamma=4 C(q, n)(\cos (\pi / n)+\cosh u)^{2}
\end{array}\right.
$$


where $(q, n)=1,1 \leq q<n / 2$, and $u \in \mathcal{U}$. Note that for $n=2$, we have $\beta=k^{2} \gamma-4$ and $\gamma=4 \cosh ^{2} u$. As $n \rightarrow \infty$, the curves $\beta=(k \sqrt{\gamma} \pm 2 \cos (q \pi / n))^{2}-4$ accumulate to the boundary of $\Sigma_{k}$, i.e., to the boundaries of $C_{k-1}$ and $C_{k}$ (see Figure 10 for an example of $\Sigma_{k}$ for $k=2$ ).

\section{REFERENCES}

[1] A. F. Beardon, The geometry of discrete groups, Springer-Verlag, New York-HeidelbergBerlin, 1983.

[2] A. F. Beardon, Fuchsian groups and nth roots of parabolic generators, Holomorphic functions and moduli, Vol. II (Berkeley, CA, 1986), 13-22, Math. Sci. Res. Inst. Publ., 11, 1988.

[3] D. B. A. Epstein and C. Petronio, An exposition of Poincaré's polyhedron theorem, L'Enseignement Mathématique 40 (1994), 113-170.

[4] W. Fenchel, Elementary geometry in hyperbolic space, de Gruyter Studies in Mathematics, 11. Walter de Gruyter \& Co., Berlin, 1989.

[5] F. W. Gehring, J. P. Gilman, and G. J. Martin, Kleinian groups with real parameters, Commun. Contemp. Math. 3, no. 2 (2001), 163-186.

[6] F. W. Gehring and G. J. Martin, Stability and extremality in Jørgensen's inequality, Complex Variables Theory Appl. 12 (1989), no. 1-4, 277-282.

[7] F. W. Gehring and G. J. Martin, Chebyshev polynomials and discrete groups, Proc. of the Conf. on Complex Analysis (Tianjin, 1992), 114-125, Conf. Proc. Lecture Notes Anal., I, Internat. Press, Cambridge, MA, 1994.

[8] J. Gilman and B. Maskit, An algorithm for 2-generator Fuchsian groups, Mich. Math. J. 38 (1991), no. 1, 13-32.

[9] E. Klimenko and N. Kopteva, Discreteness criteria for $\mathcal{R} \mathcal{P}$ groups, Israel J. Math. 128 (2002), 247-265.

[10] E. Klimenko and N. Kopteva, All discrete $\mathcal{R} \mathcal{P}$ groups whose generators have real traces, Int. J. Algebra Comput. 15 (2005), no. 3, 577-618.

[11] E. Klimenko and N. Kopteva, Discrete $\mathcal{R} \mathcal{P}$ groups with a parabolic generator, Sib. Math. J. 46 (2005), no. 6, 1069-1076.

[12] E. Klimenko and N. Kopteva, Two-generator Kleinian orbifolds, 2005, preprint.

[13] E. Klimenko and M. Sakuma, Two-generator discrete subgroups of $\operatorname{Isom}\left(\mathbb{H}^{2}\right)$ containing orientation-reversing elements, Geometriae Dedicata 72 (1998), 247-282.

[14] A. W. Knapp, Doubly generated Fuchsian groups, Mich. Math. J. 15 (1968), no. 3, 289-304.

[15] J. P. Matelski, The classification of discrete 2-generator subgroups of $\operatorname{PSL}(2, \mathbb{R})$, Israel J. Math. 42 (1982), no. 4, 309-317.

[16] E. B. Vinberg, Hyperbolic reflection groups, Russian Math. Surveys 40 (1985), 31-75.

Gettysburg College, Mathematics Department, 300 N. Washington St., CB 402, GetTYSBURG, PA 17325, USA

E-mail address: yklimenk@gettysburg.edu

LATP, UMR CNRS 6632, CMI, 39 Rue F. Joliot Curie, 13453 Marseille Cedex 13, FRANCE

E-mail address: kopteva@cmi.univ-mrs.fr 